\title{
Pesticide exposure and sprayer's task goals: comparison between vineyards and greenhouses.
}

\author{
Mandy Lambert ${ }^{\mathrm{a}}$, James Richardson ${ }^{\mathrm{a}, \mathrm{b}}$ and Sonia Grimbuhler ${ }^{\mathrm{a}}$ \\ ${ }^{\mathrm{a}}$ Cemagref: "Unité de recherché "technologies pour la sécurité et les performances des agroéquipements»1 rue \\ Pierre-Gilles de Gennes, CS 10030 92761, Antony Cedex. France \\ ${ }^{\mathrm{b}}$ Master d'ergonomie, Université Paris Sud XI, Bat 45291405 Orsay. France
}

\begin{abstract}
Plant protection products are used in agriculture to improve yields, but this use can cause contamination of the environment and is also likely to have adverse short and long term effects on agricultural workers. The field study took place in greenhouses and vineyards where operators are involved in high levels of pesticide spraying. The objective of this intervention was to identify factors explaining the influence of task factors on the exposure of greenhouse growers and vineyard workers. Thirteen operators were selected for detailed observations during one session of spraying. Video recordings provide counts of physical contacts between the operator and all the surrounding surfaces during the spraying operation. Both in vineyards and in greenhouses, physical and temporal constraints are the predominant factors in establishing a specific spraying procedure. Every action taken by the operator is a result of a compromise between safety, task performance and quality
\end{abstract}

Keywords: time constraint, physical workload, ergotoxicology,

\section{Introduction}

\subsection{Context}

Farmers are faced with two constraints: financial, in terms of productivity, and sanitary, related to their own health and the health of consumers. At present, the efficient production of crops is dependent on the use of pesticides. Plant protection products (PPP) are active substances that are employed to protect crops and to improve yield and quality. This protection requires action either on the biological processes of plants or on pests, to limit their presence. These products are intended to have an action on plants but may also have an effect on humans.

The health risks associated with pesticide use have only recently been taken into account [12]. Indeed, from 1976 with the first European directive regulating the use of pesticides to 1991 with the Directive governing the placing of plant protection products on the market, the regulations were covering essentially the general population i.e. Maximum
Residue Limits [12]. From 1991, regulations sought to reduce the health risk for pesticide applicators. From 2009, a framework of European community directives has governed the risk associated with pesticide use. Highlights of these new regulations relate to the stiffening of the authorizations for placing products on the market (CE Regulation 1107/2009), the obligation to set up a national inspection system for spraying equipment (Directive 2009/127/CE) and finally, the requirement to implement a national plan for reducing health and environmental risks associated with pesticides (Directive 2009/128/CE). In France, a national program, Ecophyto 2018, has been implemented with 9 themes with the aim of reducing environmental and health risks.

This study focuses on the analysis of spraying activity in greenhouses and viticulture. The two types of culture have distinct features that require an individual analysis of exposure prevention and operator protection. Greenhouses are closed environments that do not offer the same benefits and constraints to the operator that the vineyard offers in an open field en- 
vironment. These elements can explain differences in potential exposure values seen in the literature.

The study was designed to identify the impact of physical and mental constraints on the operating procedures employed by pesticide applicators and especially procedures that increased operator exposure to plant protection products. For this, spraying activity was compared in a closed environment of the greenhouse with open field environments in vineyards. Initially, those procedures in the two situations that potentially expose the operator have been identified. The spraying strategies developed by the operator and factors influencing these strategies have been determined through an activity analysis. In a second step, the results from the two types of culture were compared and the constraints affecting exposure were identified.

The levels of potential exposure during the mixing and loading phase and spraying application have been reported in greenhouses $[6,19]$. The values obtained show that during spraying, the operator is most exposed. Unlike in open field cultures where exposure was shown to be more important during the mixing/loading phase $(87 \mathrm{mg})$ than during spraying (37mg) [13].

Moreover, there are differences in contamination, between these two culture types, in the distribution of PPP on the operator's body surface. In viticulture, of the total operator contamination, glove contamination accounted for $39 \%$ when mixing and $53 \%$ when spraying [2]; and more broadly for treatment in an open environment the proportion was $64 \%$ and $57 \%$ respectively [13]. Measurements of hand contamination were also conducted during full pesticide treatment cycle; the hands are identified as the most exposed [20]. While for greenhouses, results show that the legs are the body site most exposed [16].

Several factors of exposure to PPPs, common to both cultures, were found in the literature, these factors explain the variability in exposure measurements and the distribution of exposure on different body parts. The equipment used has been shown to have an influence on the exposure of workers in greenhouses [15] and in vineyards [2, 20]. In greenhouses, sprayer characteristics that affect exposure are level of automation, jet pressure, position of the nozzles and the spraying speed. These determinants influence both the contamination level and the zones affected. In viticulture, automation and the presence or absence of a cabin during treatment were identified as determinants of pesticide exposure. The operating procedures employed have also been identified as an exposure factor in both situations The vineyard operator's position influences the level of exposure when spraying is performed without a cabin. In greenhouses the variability in operating procedures from one operator to another shows different exposed body zones [5]. Finally, other parameters that affect the level of exposure include the product used [2], and the weather [1]. These factors are, for the most part, not dependent on the operator and show the current limit to establishing a link between exposure and interaction with the operator's working environment.

A few studies have focused on ergonomics to demonstrate the link between levels of exposure inagriculture and the work activity itself [10]. Thus, a number of practices, said to be at risk, have been determined. Factors shown to increase the risk of exposure are: the presence of an additional physical stress [18], denial of risk [10], the negative representation of the personal protection equipment and the perception of over-protection [10].

However, the populations studied are not sufficiently representative to make generalizations about current practices. In addition, the results are mostly qualitative; more quantitative studies should be conducted in order to validate the results presented above.

\subsection{Effects of plant protection products on health}

The health effects of PPP on the operators can be classified into two groups short-term or acute effects and long-term or chronic effects.

Currently, the acute effects are well understood. These effects are usually the result of high exposure where reactions occur soon after exposure to a massive dose. The severity of effects is more or less proportional to the toxicity of the active molecule. Symptoms depend on the toxicological profile of the molecule and the route of entry into the organism. Contact with the PPP often leads to skin irritation but acute poisoning reactions may also occur in the respiratory, digestive and neurological systems [7].

Chronic effects are subject to more reserve. These effects result from a low, but prolonged, exposure repeated over time. Certain rare cancers, neurological disorders and disorders of the reproductive system have been shown to be associated with this type of exposure.

Epidemiological studies have examined the differences in cancer mortality among the farming and the general population and have shown an underrepresentation of the majority of cancers in the farming population [4]. However, some rare cancers are 
overrepresented such as skin cancer and brain. Different pathologies in connection with reproductive disorders, such as congenital malformations, have been identified [17]. Finally, studies in France on the onset of neurological disorders in the agricultural population have recently shown a significant association between Parkinson's disease and exposure to pesticides [8].

\section{Methods}

Data were collected from an activity analysis of farm workers during the different phases associated with spraying in vineyards and in greenhouses. The operators participated in a structured interview on their work practices.

\subsection{Participants}

The observations took place over two campaigns of pesticide treatment. Thirteen operators were observed in all, seven vineyard workers and five greenhouse growers. All the participants volunteered to take part in the study.

\subsection{Measures of exposure}

For this study, exposure was assessed by counting the contacts made between the operator and work environment surfaces. This method was chosen for two reasons: it has been shown that the dermal route is the main pathway of contamination [1], and that a large number of equipment surfaces are already contaminated before the start of spraying activity [10]. The purpose of this quantification is to compare pesticide exposure following the different procedures employed during the phases of mixing, loading and spraying in the two types of crop culture.

\subsection{Structured interview}

Each participant completed a structured interview at the end of spraying session. The questions were broken down into parts the first part on the characteristics of the operator, a second on the organization of business and then a final section on technical factors. The interview was designed to highlight the task and environmental constraints that were known to the operator, and were, in part, at the origin of actions that resulted in exposure to the PPP mix.

\section{Results}

\subsection{The effects of physical workload on operator exposure}

\subsubsection{Common factors}

The observations in greenhouses and vineyards showed an increase in exposure contacts over the day. There is an increase in number of contacts for successive phases over time in greenhouses (Figure 1a) and vineyards (Figure 1b). This observation was made on different phases of successive applications in different greenhouses. The spraying phase was selected as this was the only one to be repeated several times during all the observations. In viticulture, contacts were observed during mixing/loading as it is the only phase that requires a manual activity on the part of operators and is repeated during a treatment.

\subsubsection{Differences between plant cultures}

In viticulture, task procedures were modified following repeated actions by the operator related to container handling. Several task procedures involved in opening, pouring and rinsing containers changed from session to session.

Figure 2 shows that as the application process progresses the operator gradually adopts an upright stance. This change in posture is accompanied by a potential increase in exposure for the operator. The operator rests the container, on an edge measuring about $5 \mathrm{~cm}$, in the case where the container loses balance the operator may be exposed to the highly concentrated toxic product spilling from the open can.

In Figure 3a, the operator pours the first bottle in a fixed position at arm's length. During filling the second container, the operator pours the can while supporting it on the reservoir (Figure 3b). This position is inappropriate for rinsing; the operator adopts adifferent posture (Figure 3C). This posture, pressing down on the bottle that has been previously rinsed, with the hand positioned just above the can opening results in a higher exposure potential as he can come into direct contact with the PPP. 
a)

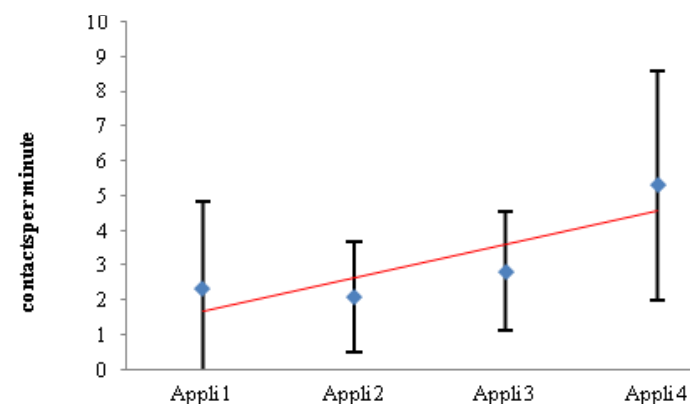

b)

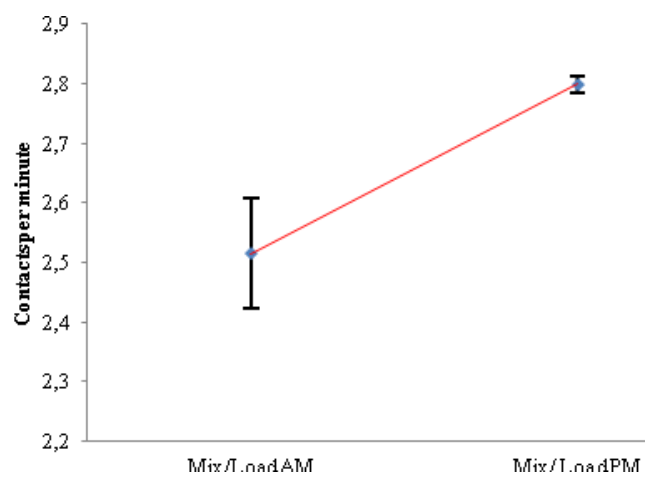

Fig. 1: Change in contacts over successive phases, $a:$ In greenhouse, $b:$ In vineyard

a)

b)

c)

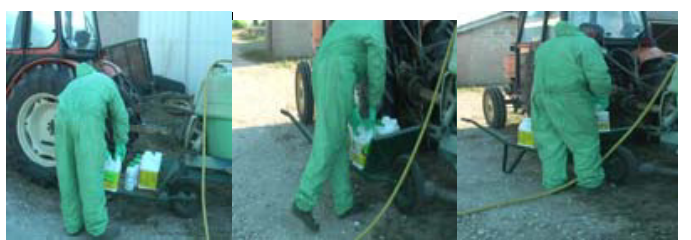

Fig. $\mathrm{n}^{\circ} 2$ : Evolution of postures when opening containers. a: Opening $1^{\text {st }}$ container, b: Opening $2^{\text {nd }}$ container, c: Opening $3^{\text {rd }}$ container.

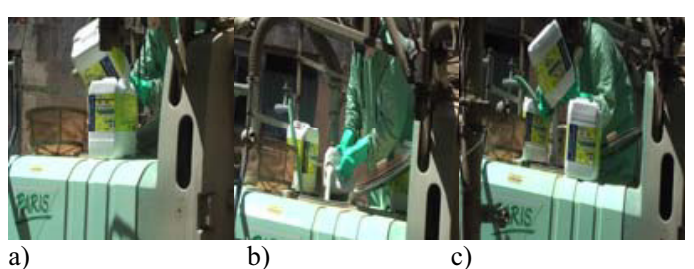

Fig. 3: Evolution oYf postures when loading / rinsing plant protection products in the sprayer reservoir. a: loading using $1^{\text {st }}$ container, b: loading using $2^{\text {nd }}$ container, c: loading using $3^{\text {rd }}$ container.

However, this observation is appropriate when using a can opening not adapted for rinsing. The observation of a similar practice when using a bottle rinse attachment allows the operator to put into place a procedure that reduces potential exposure (Figure 4).

In greenhouses, other environmental factors have been identified that are not present in vineyards, including carrying out manual spraying in an enclosed space where the thermal conditions can cause a sharp increase in physical constraint not observed in viticulture. Indeed, vineyard spraying is mostly automated and in the open field.

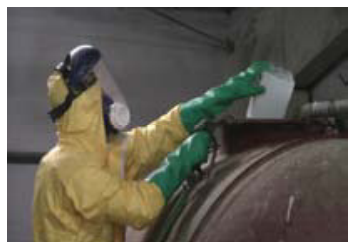

Fig. 4 : Evolution of postures when loading / rinsing plant protection products in the sprayer reservoir using a rinsing attachment.

During application two types of movement have been observed, a 'best practice' movement that ensures spraying quality and diminishes exposure and a compensatory movement, which the operators characterize as 'restful' but increases the possibility of contact with the pesticide mix as the spray head is closer to the body.

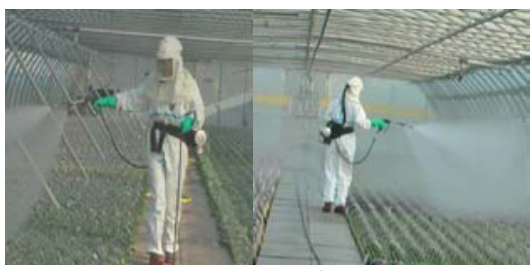

a)

b)

Fig. 5: Changes in posture during spraying in greenhouses. a: best practice gestures, b: compensatory gesture

Best practice movements (Figure 5a) can be seen when the operator is in profile, he sprays with the right hand on the right side, the upper arm is perpendicular to the trunk and performs a flexion / extension at the elbow. The distance between the nozzle and the operator's body is at its maximum when the arm is extended. The operator therefore minimizes the risk of exposure to the spray.

Compensatory movements (Figure $5 b$ ) can be described where the operator's upper arm is along the 
body with the forearm flexed at $90^{\circ}$ and he carries out the spraying with an external rotation movement, associated with rotations of hips. In this case, the operator faces the spray mist and is subject to pesticide exposure.

The operators do not perform the two types of movement routinely; the type of movement varies depending on the level of a physical workload, operator's physical capacity or operator experience. Operating procedures adopted by two operators were compared; one who alternated between two types of movement (operator A) and one who used only best practice movements (operator B) both working in identical greenhouses with the same type of sprayer.

Figure 6 shows that, during application, operator A makes more contacts with surfaces in the work environment. In addition, he is subject to increased risk of exposure by using the compensatory movements. So, Operator A adopts procedures that preserve him less from exposure than operator B who does not use compensatory gestures (Figure 6).

\subsection{The effects of time pressure on exposure}

Operators, in salaried employment, feel that time pressure is an important factor, as they are responsible for numerous tasks of which pesticide spraying is only one. If there are any delays in the spraying process, due to interruptions or breakdowns, there is a risk of losing all or part of a harvest. Here, we were interested in steps that precede, and those that follow, an incident to see the effect of time wasted on the task procedures implemented by operators. The differences in number of exposure contacts made by the operators are shown in Figure 7.The greenhouse operator makes twice the number of contacts with the contaminated environment following an incident than during a phase without incident. Time delays can provoke a non-respect of task goals if the operator does not find a suitable response. In the case observed here, the operator changed his procedures in order to catch up lost time (Figure 7B), but in return he performs procedures that increase potential exposure to PPPs. However, the presence of an incident did not result in increased number of contacts for the vineyard worker.

\subsection{Structured interviews}

Following the observations, each operator participated in a structured interview to understand their reasons for adopting different procedures. Interviews with greenhouse workers have highlighted the physical and organizational pressure exerted on them. Indeed, some operators consider the best practice gesture painful; these are the same operators who also adopt compensatory movements.

Operators also remarked that ambient temperature in the greenhouse increased over the day which contributed to physiological load and affected spraying performance. At the same time greenhouse operators are subject to organizational constraints imposed on them by their superiors. Pesticide spraying often takes place on Saturday, to ensure safe re-entry conditions, and has to be completed by the end of the morning.

In viticulture, some operators have explained that suffering from back pain when opening a pesticide container encourages adopting postures that are less painful but puts them at higher risk of exposure. Time pressure is not an important factor remarked on by vineyard workers.

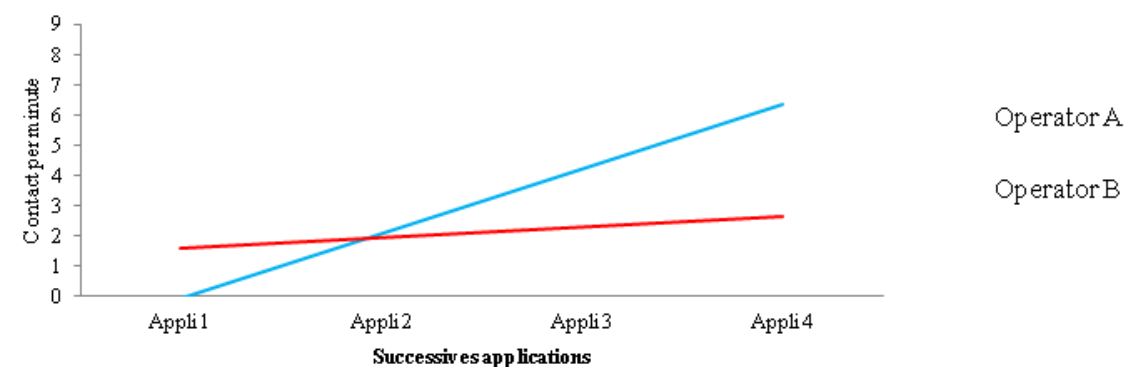

Fig. 6: Changes in contacts during spraying as a function successive applications 


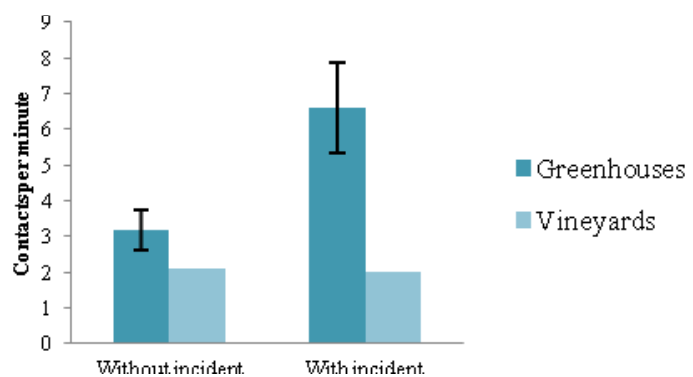

b)

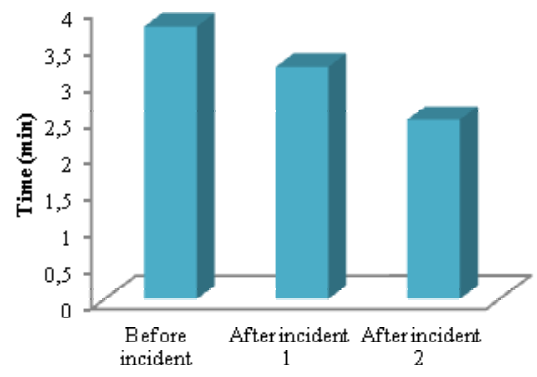

Fig. 7 a : Comparison of number contacts in greenhouse and vineyards following or not an incident. b: Duration of phases before and following an incident

Indeed, all the vineyard operators are owners and believe that spraying has a very high priority.

In both types of culture, according to the operators thermal discomfort due to wearing personal protective equipment in all or certain specific phases is a significant factor in the development of fatigue and subsequently risk of exposure.

\section{Discussion}

The increased number contacts between application phases and responses during interviews shows a link between the physical discomfort felt by the operators and increased exposure. When ambient temperature increases, coupled with wearing personal protective equipment, operators make more contacts with contaminated surfaces during the two phases studied. Operators make a compromise between objectives, one of avoiding fatigue, which can be unfavorable in terms of exposure, and the other of safeguarding health.

The surface area to be treated and the farm size are two main characteristics that differentiate the two types of culture. For the farms observed in this study, the average farm size for greenhouse growing is 16 ha while for vineyards the average is $25 \mathrm{ha}$. The application environments are also very different; a greenhouse is enclosed, which explains the difference in equipment used in these two cultures. A 2009 study [3] showed that $76 \%$ of greenhouse growers in France use a backpack sprayer with a lance or atomizer. In vineyards, a 2010 survey (personal communication, SUI, 2010) shows that $87 \%$ of farmers use a tractor with a cab, the proportion is $70 \%$ is if one considers pressurized cabs. In viticulture, the operator, on a tractor, is a distance of several meters $( \pm 3 \mathrm{~m})$ away from the spray nozzles.

In viticulture, repeated heavy lifting influences the task procedures adopted by operators. This factor is related to the farm size that usually corresponds to the entire surface treated with pesticides. The average farm size is smaller for greenhouse growers, and the quantity of PPP used to fill the reservoir is smaller so, in principle, greenhouse operators handle lighter loads of concentrate. While in viticulture, certain products are still frequently conditioned in heavy units of up to $25 \mathrm{~kg}$ or $20 \mathrm{~L}$. This may explain the differences observed in procedures for handling containers. Heavy lifting and maintaining a static posture can be the source of physical load that results here in changing task procedures to safeguard the operator from discomfort but in turn, this can be a source of excess exposure. Indeed, observations show that the use of a can rinsing attachment allows the operator to adopt task procedures that do not lead to additional exposure.

The existence of back pain in vineyard workers is also a possible explanation for changes in the observed procedures. Such a pain induces a restriction in the freedom of movement. The operator performs the procedures producing least pain that are not necessarily the best suited for protection against PPP exposure in the work situation.

In greenhouses, spraying is carried out manually; operators have highlighted the difficulty of always making the same gestures that can cause discomfort. In addition, they are obliged to continue spraying activity due to the time constraints exerted on them. The change in spraying gestures observed during pesticide application in greenhouses can be explained by a compromise made by the operator. This compromise gives priority to respecting time constraints 
and reducing physical load at the expense of safeguarding the operator from exposure to PPP.

In this study time pressure has been shown to be an influence on task activity for employees who have limited flexibility in their work procedures.

In greenhouses, it seems that the most difficult phase for the operator is pesticide application. While in viticulture, it is essentially the mixing and loading phase. These results are similar to those obtained in the literature $[19,13]$.

Physical and temporal constraints are responsible for compromises in the operator's task objectives that can influence exposure by modifying task procedures. The task procedures adopted by operators are the result of a compromise between health, production and quality objectives [11]. Following changes over time in task activity, objectives or goals may change and lead to situations where the task constraints become too stringent, and the resulting activity may lead to over-exposure for the operator [9].

The operator then puts in place a system for regulating activity in order to optimize the effects on the operator or on a task [14]. Spérandio [9] has demonstrated, in other work situations, that operators change their task procedures as a function of workload. The results revealed that operators employed a directory of procedures and chose a particular procedure according to the level of requirements of the task. When the level of demand or perceived workload is higher than that acceptable, the operator develops task procedures that are less costly. Similar modifications of task procedures have been shown in this study. Operators change their procedures to make them more cost effective but by doing so can increase their risk of exposure to PPP.

When the goals change, procedures evolve, and the activity necessary to complete the task cannot be anticipated. Each operator then creates new strategies of action with reference to his own mental representations, which explains why exposure varies from one operator to another. In other words, thinking about his actions allows the operator, during task execution, to avoid making new gestures that are a potential risk of exposure.

\section{Conclusion}

There are a number of limits to the methodology used in this study. A major limitation concerns the assessment of contact by observation, used to determine the sources of exposure, which do not provide a real measure of operator exposure. Moreover, the distinctions between short light touch events and prolonged heavy touch events have not been taken into account, in viticulture long contacts are observed during mixing/loading while in the greenhouse long contacts take place mainly during spraying.

This exploratory study has demonstrated that task objectives and task procedures are at the centre of an active regulation by the operator. The current training provided to greenhouse growers is mainly based on the notion of danger of exposure to PPPs, which may encourage the sprayers not to take an active role in avoiding risks. Furthermore, the identification of different factors that are sources of exposure reflects the specificity of the two types of plant culture. Training for exposure prevention programs will only be effective on condition that, in the materiel used for the program, the operators can identify with work situations that they are used to.

Future work will involve the development of metrology to quantify accurately the impact of factors on operator exposure. In addition, the impact of workload on the operator's task patterns as well as other factors, such as the influence of sprayer type, which have been highlighted in a qualitative way here, will be investigated.

\section{References}

[1] C. Aprea, L. Centi, S. Santini, L. Lunghini, B. Blanchi and G. Sciarra, Exposure to omethoate during stapling of ornamental plants in intensive cultivation tunnels: influence or environmental conditions on absorption of the pesticide, Archives of Environmental Contamination and Toxicology 49 (2005), 577-588.

[2] I. Baldi, P. Lebailly, S ; Jean, L. Rougelet, S. Dulaurent and P. Marquet, Pesticide contamination of workers in vineyards in France, Journal of Exposure Science and Environmental Epidemiology 16 (2006), 115-124.

[3] H. Beyer and S. Grimbuhler, Operator actual protection against pesticides exposure in French greenhouses, International Conference on Agricultural Engineering, ClermontFerrand, 2010.

[4] A. Blair, S.H. Zahm, N.E. Pearce, E.F. Heineman and J.F. Fraumeni, Clues to cancer etiology from studies of farmers, Scandinavian Journal of Work, Environmental \& Health 18 (1992), 209-215.

[5] S.M.F. Calumpang, Exposure of four Filipino farmers to parathion-methyl while spraying string beans, Pesticide Science 46 (1996), 93-102.

[6] E. Capri, R. Alberici, C.R. Glass, G. Minuto and M. Trevisan, Potential operator exposure to procymidone in greenhouses, Journal of Agricultural and Food Chemistry 47 (1999), 44434449.

[7] S. Duret, Le risque phytosanitaire en milieu agricole. A travers le cas des maraichers/horticulteurs de Haute Savoie, Mé- 
moire de Maitrise. Université PaulValéry, Montpellier III, 2006.

[8] A. Elbaz, J. Clavel, P.J. Rathouz, F. Moisan, J.P. Galanaud, B. Delmotte, A. Alpérovitch and C. Tzourio, Professional exposure to pesticides and parkinson disease, Annals of Neurology 66 (2009), 494-504.

[9] P. Falzon and C. Sauvagnac, Charge de travail et stress, P. Falzon ed., Puf, Paris, 2004, pp.175-190.

[10] A. Garrigou, I. Baldi, P. Le Frious, R. Anselm and M. Vallier, Ergonomics contribution to chemical risks prevention: an ergotoxicological investigation of the effectiveness of coverall against plant pest risk in viticulture, Applied Ergonomics 42(2011), 321-330.

[11]F. Guerin, A. Laville, F. Daniellou, J. Duraffourg and A. Kerguelen, Comprendre le travail pour le transformer, Eds, Réseau éditions ANACT, Lyon.

[12]A.J. Karabelas, K.V. Plakas, E.S. Solomou, V. Drossou and D.A. Sarigiannis, Impact of European legislation on marketed pesticides - A view from the standpoint of health impact assessment studies, Environmental International, 35 (2005), 1096-1107.

[13]P. Lebailly, V. Bouchart, I. Baldi, Y. Lecluse, N. Heutte, A. Gislard and J.P. Malas, Exposure to pesticides in open field farming in France, The Annals of Occupational Hygiene 53(2009), 69-81.

[14]J. Leplat, La notion de régulation dans l'analyse de l'activité, Pistes 8 (2006)

[15]D. Nuyttens, P. Braekman, S. Windey and B. Sonck, Potential dermal pesticide exposure affected by greenhouse spray appli- cation technique, Pest Management Science 65 (2009), 781790.

[16]L.M. Ramos, G.A. Querejeta, A.P. Flores, E.A. Hughes, A. Zalts and J.M. Montserrat, Potential dermal exposure in greenhouses for manual sprayers : Analysis of the mix/load, application and re-entry stages, Science of the Total Environment 408 (2010), 4062-4068.

[17]R.P. Rull, B. Ritz and G.M. Shaw, Neural tube defects and maternal residential proximity to agricultural pesticides applications, American Journal of Epidemiology 163 (2006), 743753.

[18]L.I. Sznelwar, Analyse ergonomique de l'exposition des travailleurs agricoles aux pesticides, Essai d'ergotoxicologique, Ph.D. Dissertation, Conservatoire National des Arts et Métiers Paris, 1992.

[19]A. Tuomainen, M. Mäkinen, R. Glasse and R. Kangas, Potential exposure to pesticides in Nordic greenhouses, Bulletin of Environmental Contamination and Toxicology 69 (2002), 342-349.

[20]M. Vitali, C. Protano, A. Del Monte, F. Ensabella and M. Guidotti, Operative modalities and exposure to pesticides during open field treatments among a group of agricultural subcontractors, Archives of Environmental Contamination and Toxicology 57 (2008), 193-202.

SUI personal communication - M. Grandjean 2010, Safe Use Initiative. France 